\title{
A new method for determination of tert-butylhydroquinone (TBHQ) in RBD palm olein with FTIR spectroscopy
}

\begin{abstract}
Fourier transform infrared (FTIR) spectra of refined bleached and deodorized (RBD) palm olein samples between 3600 and $2800 \mathrm{~cm}-1$ were used for quantitative determination of the content of tert-butylhydroquinone (TBHQ). The method was based on sodium chloride $(\mathrm{NaCl})$ windows with transmission path fixed at $50 \mu \mathrm{m}$ at room temperature. Fifty stripped oil samples spiked with known amounts of TBHQ concentrations up to $300 \mathrm{mg} / \mathrm{kg}$ (ppm) were separated into two sets for calibration and validation models based on partial least squares (PLS) analyses. The accuracy of the method was comparable to that of the method established by the International Union of Pure and Applied Chemistry (IUPAC) with a coefficient of determination (R2) of 0.9961 and standard error of calibration of (SEC) 5.06. The model was validated, and the $\mathrm{R} 2$ of validation and standard error of prediction were 0.9951 and 6.49 , respectively. The standard deviation of difference for repeatability for the method was comparable to that for the IUPAC method. The new FTIR method developed for determining TBHQ in palm olein was demonstrated to be efficient, accurate, rapid and required minimum solvent as only acetone was used for cleaning $\mathrm{NaCl}$ windows. The method is suitable for routine quality control analysis. (C) Copyright 2004, Blackwell Publishing.
\end{abstract}

Keyword: Oil samples; Palm olein; Partial least squares (PLS); Refined bleached and deodorized (RBD); Fourier transform infrared spectroscopy; Ketones; Mathematical models; Oils and fats; Quality control; Separation; Sodium chloride; Solvents 